\title{
Review Article \\ Diagnostic Options and Challenges for Dengue and Chikungunya Viruses
}

\author{
Stacey K. Mardekian and Amity L. Roberts \\ Sidney Kimmel Medical College at Thomas Jefferson University, 117 South 11th Street, PAV 207, Philadelphia, PA 19107, USA \\ Correspondence should be addressed to Amity L. Roberts; amity.roberts@jefferson.edu
}

Received 10 March 2015; Revised 23 June 2015; Accepted 25 June 2015

Academic Editor: Marcus Lacerda

Copyright ( $) 2015$ S. K. Mardekian and A. L. Roberts. This is an open access article distributed under the Creative Commons Attribution License, which permits unrestricted use, distribution, and reproduction in any medium, provided the original work is properly cited.

\begin{abstract}
Dengue virus (DENV) and Chikungunya virus (CHIKV) are arboviruses that share the same Aedes mosquito vectors and thus overlap in their endemic areas. These two viruses also cause similar clinical presentations, especially in the initial stages of infection, with neither virus possessing any specific distinguishing clinical features. Because the outcomes and management strategies for these two viruses are vastly different, early and accurate diagnosis is imperative. Diagnosis is also important for surveillance, outbreak control, and research related to vaccine and drug development. Available diagnostic tests are aimed at detection of the virus, its antigenic components, or the host immune antibody response. In this review, we describe the recent progress and continued challenges related to the diagnosis of DENV and CHIKV infections.
\end{abstract}

\section{Introduction}

Dengue virus (DENV) and Chikungunya virus (CHIKV) are single-stranded, positive-sense RNA viruses. DENV belongs to the family Flaviviridae and genus Flavivirus of which there are 5 known serotypes (DENV1-5). CHIKV belongs to the family Togaviridae and genus Alphavirus of which there are 3 known strains (Asian-West African; East-Central; South African) [1]. The genome of each virus is approximately $11 \mathrm{~kb}$ in length $[1,2]$. The DENV genome encodes three structural (C, prM, and E) and seven nonstructural (NS1, NS2B, NS3, NS4A, NS4B, and NS5) proteins [3]. The CHIKV genome encodes three structural $(\mathrm{C}, \mathrm{E} 1$, and E2) and four nonstructural (nsP1-4) proteins [1].

Both viruses are arthropod-borne viruses (arboviruses) sharing a common vector: mosquitos of the Aedes genus, specifically $A$. aegypti and A. albopictus [4]. Both viruses circulate in similar geographic regions. In nonendemic regions, travel-associated infections are an important consideration for patients with a recent travel history who present with fever. Concurrent infection with both viruses, transmitted from either two different mosquitos or one dually infected mosquito, is possible $[5,6]$. For DENV, transmission has also been reported to occur via infected blood products, organ donation, and prenatal and/or perinatal vertical transmission [7].

While DENV and CHIKV present similarly as an acute febrile illness, these two viruses have vastly different management strategies and outcomes. The majority of CHIKV infections are self-limiting with chronic joint disease being the most common long-term outcome, and fatality is exceedingly rare. Nonsteroidal anti-inflammatory drugs (NSAIDs) are the mainstay treatment for CHIKV, but NSAIDs should be avoided until DENV is confidently ruled out, as NSAIDs are contraindicated in DENV infection [8]. DENV is likewise commonly a self-limiting illness, yet this diagnosis necessitates stricter monitoring due to the potential for more significant morbidity and mortality. Infection with one serotype of DENV confers lifelong immunity to that particular serotype but only short-term immunity to the other serotypes; subsequent infections with a different serotype increase the risk of severe complications [7].

\section{Epidemiology}

The majority of DENV and CHIKV infections affect people residing in endemic areas, which include most of the tropical 
and subtropical regions in the world. Many of these areas serve as popular tourist destinations and, consequently, dengue-related infections have recently surpassed malaria and gastrointestinal infections as the most common cause of fever among travelers [23]. The major endemic regions include Southeast Asia, the Western Pacific, the Eastern Mediterranean, Africa, and the Americas [9]. Specific countries with cocirculation and coinfections of DENV and CHIKV include India, Sri Lanka, Gabon, Cameroon, Madagascar, Indonesia, Singapore, and Thailand [24]. In the United States, autochthonous outbreaks of DENV have been reported in Hawaii and along the Texas-Mexico border, and outbreaks of both DENV and CHIKV have recently occurred in southwest Florida $[6,25]$.

\section{Clinical Presentation}

These two viruses share a similar geographic distribution; unfortunately, their clinical manifestations also show substantial overlap. The typical incubation periods for DENV and CHIKV are 4-7 days and 3-7 days, respectively [4]. Patients infected with either virus typically present with acute onset of fever, myalgia, and headache, and some patients experience a maculopapular rash and/or gastrointestinal symptoms $[4,6]$.

A classification scheme for DENV, put forth by the World Health Organization (WHO) in 2009, includes criteria for probable dengue and severe dengue [9]. Most DENV infections are either asymptomatic or mild and self-limited, but there are "warning signs" that may suggest which patients may progress to severe disease and require stricter medical management [9]. Severe dengue may manifest as significant plasma leakage, hemorrhagic complications, and/or severe organ impairment, so early recognition of DENV infection is imperative [9]. Compromising the sensitivity of the WHO classification scheme is the fact that patient age influences the type and severity of symptoms; Low et al. found that fewer older adults reported symptoms of myalgia and arthralgia, as well as mucosal bleeding, which is one of the primary "warning signs" [26].

The clinical course for CHIKV is likewise typically mild and self-limited. The hallmark presentation of CHIKV is a bilateral migratory arthralgia, often intense, affecting mainly the small joints of the extremities $[1,4]$. However, most children with CHIKV report only mild arthralgia [8]. The major long-term complication is persistence of joint pain and stiffness, which may last years after resolution of the initial infection [1]. Rarely, CHIKV infection is associated with neurologic, ophthalmologic, and hemorrhagic disease $[4,5]$.

While neither infection possesses a defining clinical feature, there are suggested trends in the symptomatology and complete blood count $(\mathrm{CBC})$ results that may help differentiate between the two infectious processes. It is suggested that, at initial presentation, significantly more DENV patients have thrombocytopenia (platelets $<100 \times$ $10^{9} / \mathrm{L}$ ) and associated minor bleeding complications such as petechiae and nose bleeds, while patients with CHIKV are more likely to have arthralgia. Leukopenia is common to both infections at initial presentation but tends to be more pronounced in DENV patients; CHIKV patients tend to have higher white blood cell (WBC) counts $\left(>3.6\right.$ or $5.0 \times 10^{9} / \mathrm{L}$ according to two separate authors) than DENV patients $[4$, $6,8]$. During the course of illness, DENV patients are more likely to have abdominal pain and the $\mathrm{CBC}$ will demonstrate leukopenia, neutropenia, and thrombocytopenia that is more frequent and more pronounced than in CHIKV patients. In contrast, CHIKV patients may show a shorter duration of fever, conjunctivitis, acute arthritis, and more prominent arthralgia affecting multiple joints [6]. While these trends in clinical findings may be helpful, they are neither specific nor consistent enough to be considered diagnostic.

Unfortunately, there is no single clinical or laboratory marker available for distinguishing DENV or CHIKV infection from each other or from other acute febrile illnesses. Therefore, both of these viruses must be initially included in the differential diagnosis for a patient with suspicious clinical symptoms who is living in or returning from travel to an endemic area. Clinical features can serve, at best, as a guide for favoring one virus over the other, as patients may present atypically, either by lacking the "classic" signs or symptoms as mentioned above, or by presenting in an uncharacteristic manner. Laboratory diagnostic tests are thus essential for accurate identification of the causative virus.

\section{Methods for Diagnosis}

A wide variety of laboratory diagnostic methods are available to aid in the diagnosis of DENV and CHIKV infections. The premise of these tests is detection of the virus, viral components (antigens or nucleic acid), or the host immunologic response to the virus [10]. Therefore, selection and interpretation of testing depends on the kinetics of viremia and antibody response, which differ between primary and secondary infections. Other factors influencing test choice include the purpose of testing and availability of resources. Each type of test offers unique advantages and disadvantages, and a combination of tests may be employed in order to increase diagnostic confidence. For a summary of available tests for DENV and CHIKV infection, see Tables 1 and 2, respectively.

4.1. Overview of Currently Available Tests. The acute febrile phase of infection corresponds to the period of viremia, which lasts typically from 5 days after onset of fever for both DENV and CHIKV. During this time, diagnosis rests on isolation of the virus, viral RNA, or viral antigen from the specimen. Isolation of DENV or CHIKV can be performed via mosquito inoculation or cell culture; CHIKV isolation can also be accomplished by intracerebral inoculation of mice [16]. Virus may be recovered from serum, plasma, whole blood, or tissues collected at autopsy. Mosquito inoculation is the most sensitive isolation method but is impractical for routine diagnosis due to the highly specialized requirements and high maintenance costs [3]. Cell culture is in wider use, with preference given to the mosquito cell line C6/36 (cloned 


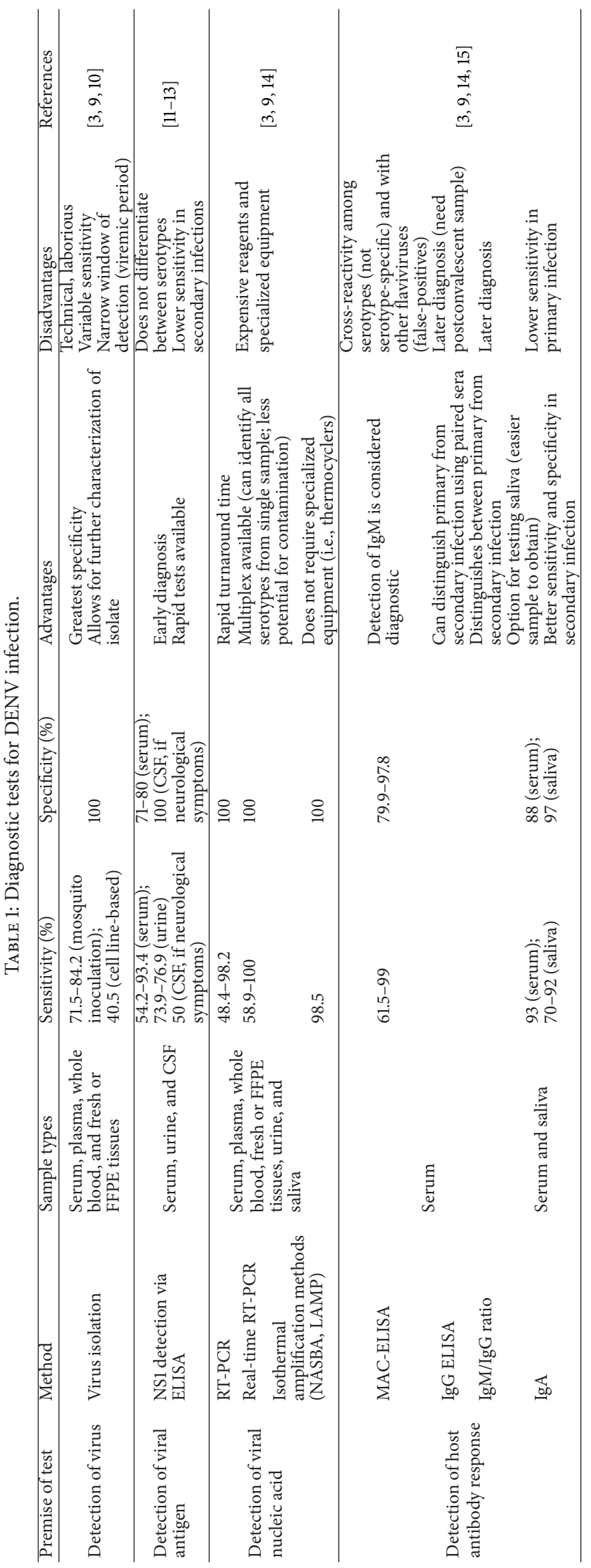




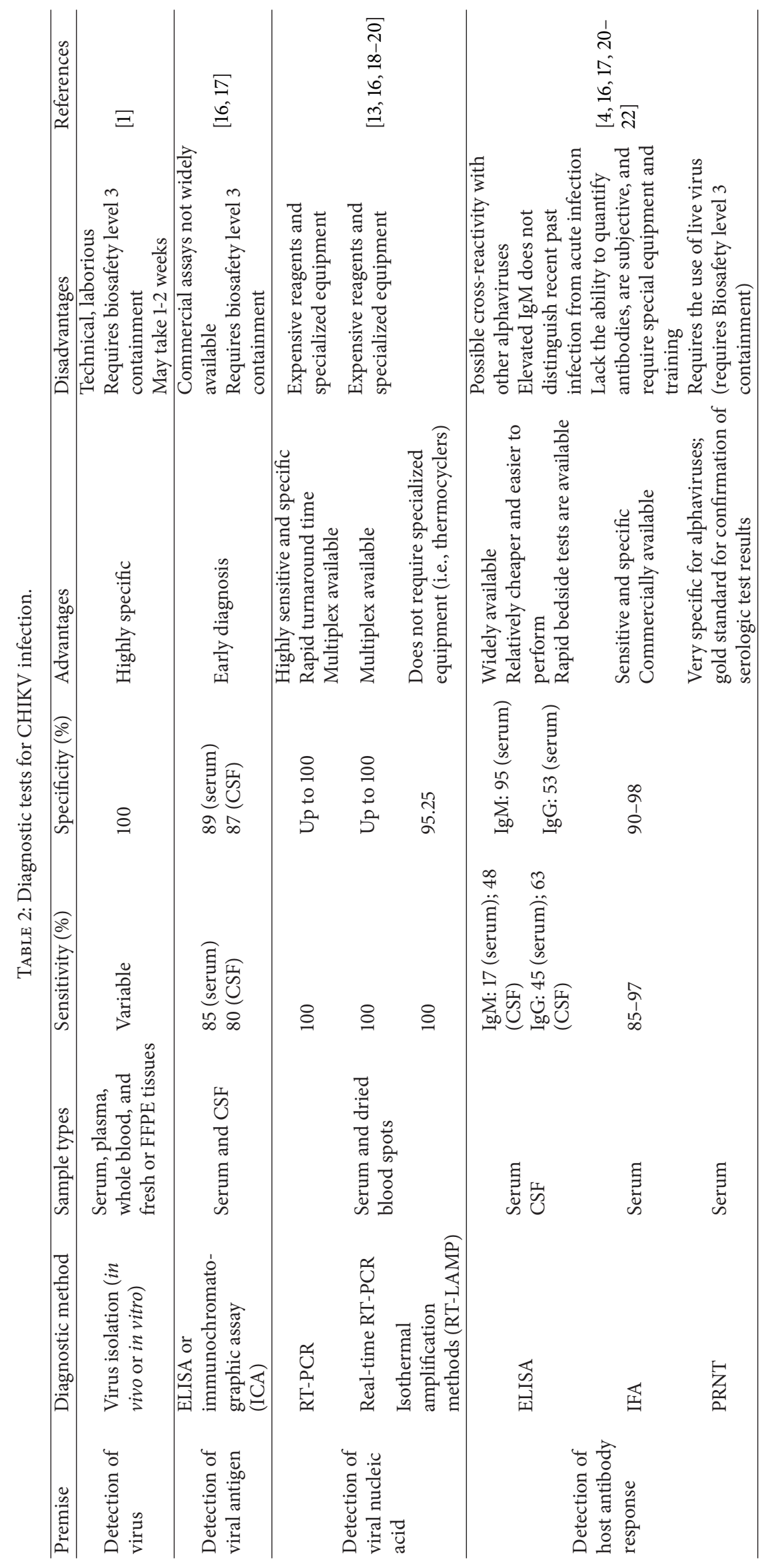


from A. albopictus) or AP61 (cloned from A. pseudoscutellaris) $[9,16]$. Other less sensitive options include mammalian cell cultures such as Vero, LLC-MK2, and BHK-21 [3]. The resultant virus isolate may be further characterized during subsequent in vitro studies, such as genome sequencing, virus neutralization, and infection studies [3]. Virus isolation is highly specific and has a theoretical detection limit of a single viable virus, although, in practice, the sensitivity is only approximately $40.5 \%$ in cell line-based virus isolation. It also requires highly trained operators, a dependence on sample integrity and a short viremia period, thus providing a narrow window of opportunity from illness onset. Virus isolation followed by an immunofluorescence assay for confirmation requires days to weeks $[9,16]$. Therefore, despite its advantages, this approach is not widely used in routine diagnostic laboratories and may serve more use in surveillance purposes. A more recent development in viral isolation is described by Patramool et al., who used anionic polymer-coated beads to isolate DENV and CHIKV [27]. This may prove a useful strategy to monitor the status of circulating mosquitos in regions at risk for outbreaks with these arboviruses. Compared to traditional isolation techniques, this method provides reduced cost, good sensitivity, and rapidity, which is conducive to simultaneous analysis of a large number of samples [27].

Compared to virus isolation, viral nucleic acid detection techniques performed on acute-phase specimens offer better sensitivity with a much more rapid turnaround time. Viral nucleic acid can be detected for a few additional days beyond the period of viremia. Detection of viral nucleic acid can be accomplished by reverse transcriptase polymerase chain reaction (RT-PCR), real time RT-PCR, or isothermal amplification methods. All of these methods involve three basic steps: viral RNA extraction, amplification, and detection and characterization of the amplified product [9]. There is a wide variety of specimen types that can be tested with RT-PCR, including blood, serum, plasma, and fresh or formalin-fixed paraffin-embedded tissues. For DENV, urine and saliva have been found to be suitable specimen types as well [3]. Testing urine samples by real-time RT-PCR provides a larger window of detection that extends well past the viremia period; DENV RNA may be detected in urine up to day 16 , compared to day 8 for blood specimens [28]. The ability to test urine and saliva is advantageous in patients for whom blood samples are difficult to obtain, such as in newborns and patients with hemorrhagic syndromes [14].

RT-PCR using primers designed for structural and nonstructural domains has been found to be useful in the rapid diagnosis of CHIKV. The combination of RT-PCR/nested PCR has proved efficient for specific detection and genotyping of CHIKV. Loop-mediated isothermal amplification (LAMP) assays can be rapidly carried out at a single temperature in a water bath, with visually detectable results, and comparable sensitivities to conventional PCR [17].

Detection of viral antigens is another diagnostic methodology available for DENV infection. Nonstructural protein 1 (NS1) antigen is a highly conserved glycoprotein produced during the virus replication process, and a soluble form of NS1 accumulates in high concentrations in the serum of patients with both primary and secondary DENV infections $[29,30]$. Several commercial assays, consisting of both rapid tests and enzyme-linked immunosorbent assay (ELISA) kits, are available for the detection of the NS1 antigen. Serum is the most common sample type. DENV NS1 can also be detected in urine samples during the acute phase of DENV infection, which provides an opportunity for the development of a rapid noninvasive test [11]. Lastly, NS1 antigen may be detected in the cerebrospinal fluid (CSF) of patients with neurological symptoms [12]. A downfall is that these tests do not differentiate between dengue serotypes, as NS1 is highly conserved by all serotypes. Additionally, these tests are most successful during the acute phase of illness and lose sensitivity once the period of viremia ends. The sensitivity of NS1 has also been found to be lower in DENV secondary infections, which is thought to be due to assay interference by anti-NS1 antibodies which are present more frequently in secondary infections $[10,29]$. An antigenbased commercial detection assay is not widely available for $\mathrm{CHIKV}$, and the ones described thus far in the literature have unclearly established performance characteristics [21, 22].

After the period of viremia, the methods described thus far become much less sensitive for diagnosis. At this point, the best diagnostic strategy entails detection of antibodies indicative of host immune response to the virus. However, the caveat is that individuals in endemic areas often have immunologic levels to these viruses. Serologic methods include ELISA, indirect immunofluorescence assays (IFA), hemagglutination inhibition (HI), and microneutralization (MNt) [1]. ELISA and IFA are rapid and sensitive techniques for detecting virus-specific antibodies and can distinguish between IgG and IgM. For techniques that cannot make this distinction (HI and $\mathrm{MNt}$ ), it is required to compare paired serum samples (acute and convalescent phases) to establish recent infection.

For DENV, serologic methods are most commonly employed, in particular IgM capture ELISA [4]. IgM antibodies are detectable in $50 \%$ by days $3-5$ after onset, $80 \%$ by day 5 , and $99 \%$ by day 10 after initial symptoms. They may persist for months; hence DENV IgM antibodies are a reliable marker of recent but not necessarily acute infection [29]. IgG antibody response develops a few days after the onset of IgM antibodies, and IgG may persist for many years [29]. Serologic confirmation of infection requires demonstration of a fourfold rise in antibody titer between acute and convalescent phase sera, or by demonstration of IgM antibodies specific for the virus [16]. Patterns of antibody response differ between primary and secondary infections, with primary dengue invoking stronger and more specific IgM response than in secondary, which have stronger and more rapid IgG response. Prior vaccination against other Flavivirus (Japanese encephalitis virus; Yellow-fever virus) or prior infection with nondengue flaviviruses (including West Nile) can potentially influence antibody responses measured in some assays [4]. The recent introduction of rapid diagnostic kits that offer combined detection of NS1 and IgM/IgG antibodies was an effort to create a point-of-care test with better performance characteristics [13]. Evaluation 
of some of these combined tests has revealed diagnostic sensitivity of $89-93 \%$ and specificity of $75-100 \%$ [3, 13].

A combination of molecular and IgM antibody detection assays is recommended for diagnosis of CHIKV infection. Some advocate adopting an algorithmic approach, wherein the IgM capture ELISA is used as an initial screening tool followed by the use of rapid molecular assays in CHIKV IgM negative samples, to facilitate rapid diagnosis during outbreaks [18].

\subsection{Simultaneous Testing for DENV and CHIKV. Because} infection with DENV and CHIKV should be on the differential diagnosis together at the initial patient presentation, tests that screen for these viruses simultaneously are preferred to test for them separately. CHIKV and DENV are not readily differentiated serologically due to cross-reactivity of their serocomplexes, so there is a reliance on molecular detection methods for this purpose [19]. A one-step duplex conventional RT-PCR assay for distinguishing DENV and CHIKV has been reported [20]. Saha et al. developed a highly sensitive and specific, rapid one-tube duplex RT-PCR assay which provides a result within 110 minutes [19]. Two authors have described a one-step multiplex real-time RT-PCR assay that can simultaneously detect and quantitate RNA for all DENV serotypes and CHIKV. Cecilia et al. report a sensitivity of $100 \%$ for DENV and $95.8 \%$ for CHIKV, while the specificity was $100 \%$ for both viruses when compared to conventional RT-PCR [24]. Pongsiri et al. report an assay sensitivity of $97.65 \%$ and specificity of $92.59 \%$ when compared to conventional RT-PCR [31]. Real-time reverse transcription-loopmediated isothermal amplification (RT-LAMP) is a sensitive alternative to real-time PCR for use in field applications [18]. A RT-LAMP method has been described in which a reverse transcription and amplification was designed in one step with two tubes under the same reaction conditions for the rapid identification and quantitative detection of RNA for CHIKV and DENV, respectively [32]. This assay has a sensitivity of $100 \%$ and specificity of $95.25 \%$. The LAMP reaction can be ended within one hour under isothermal conditions and does not require sophisticated instruments, making this method adaptive to field diagnosis. Additionally, the use of a turbidimeter allows for quantitative detection of viral load [32]. For RT-PCR assays described above, the onestep process reduces the chance of contamination and there is lack of cross-reactivity between related Flavivirus groups and DENV [19].

\subsection{Sending Out Samples. Within the United States, CHIKV} testing is performed at the Centers for Disease Control and Prevention (CDC), a limited number of select state health departments, and one commercial laboratory. The CDC's Arbovirus Diagnostic Laboratory at the Division of VectorBorne Diseases (DVBD) is located in Fort Collins, CO. Test results are normally available 4 to 14 days after specimen receipt, but reporting times may be longer during summer months when arbovirus activity increases. Initial serological testing is performed using IgM capture ELISA and IgG ELISA. If the initial results are positive, further confirmatory testing is performed which may delay the reporting of final results. All results are sent to the appropriate state health department.

The CDC Dengue Branch, located in San Juan, Puerto Rico, provides DENV testing free of charge to submitting physicians and state and private laboratories. A "Dengue Case Investigation Form" must accompany the specimen. One potentially problematic issue with sending samples to this laboratory is that an international shipping license is required. Another challenge, especially for underdeveloped countries, is specimen preservation during shipment. The $\mathrm{CDC}$ recommendation is that the serum specimen is frozen immediately after separation and sent on dry ice, or alternatively kept refrigerated and sent in cold packs.

4.4. Future Test Developments. Other diagnostic methodologies may be available for future use in the laboratory diagnosis of DENV and CHIKV infection. One technique becoming an increasingly popular serological option in arbovirology is microsphere-based immunoassay (MIA). This technology is based on detection by flow cytometry of antigen or antibody attached to microspheres or beads. This is a much more rapid test than MAC-ELISA and also has the potential for performance in multiplex [33]. Similarly, microarray technology, which focuses on detection of nucleic acid fragments corresponding to different pathogens, is useful to screen a sample for the many pathogens on a wide differential diagnosis for infectious symptoms in a given region [10]. Finally, mass spectrometry could be applied to this field of diagnosis, proving especially useful in determining viral serotypes and genotypes during an outbreak [9].

\section{Conclusion}

Confirmation of DENV or CHIKV infection requires laboratory diagnosis. Molecular assays are more sensitive for diagnosis in the early stages of illness (2-5 days after onset) when antibodies are not detected. However, in the later stages of illness, the sensitivity of molecular methods decreases due to the onset of a brisk immune response and corresponding reduction in viral load. At this stage, the IgM ELISA is a more sensitive diagnostic test.

An ideal diagnostic test meets certain key criteria: affordability by those at risk of infection, specificity, sensitivity, ease of use, rapid results, little reliance on equipment, and delivery to those in need [29]. The ideal test should also be part of a multiplexed assay for other pathogens causing acute undifferentiated fever, such as malaria [17]. Progress for DENV and CHIKV diagnostic testing has been made. Generally, tests with high sensitivity and high specificity require more complex technologies and technical expertise, while rapid tests may sacrifice sensitivity and specificity for the advantages of speed and ease of performance. It is difficult to find a balance between accessibility of a diagnostic method and the confidence in the test results. Antigen detection assays seem most promising for rapid and early diagnosis in rural areas. In this regard, development of DENV diagnostic tests is ahead of those for CHIKV, but clearly both of these arboviruses are important causes of disease in their shared endemic regions and in travelers to these areas. 


\section{Conflict of Interests}

The authors declare that there is no conflict of interests regarding the publication of the paper.

\section{References}

[1] C. Caglioti, E. Lalle, C. Castilletti, F. Carletti, M. R. Capobianchi, and L. Bordi, "Chikungunya virus infection: an overview," New Microbiologica, vol. 36, no. 3, pp. 211-227, 2013.

[2] A. B. Sudeep and D. Parashar, "Chikungunya: an overview," Journal of Biosciences, vol. 33, no. 4, pp. 443-449, 2008.

[3] K. F. Tang and E. E. Ooi, "Diagnosis of dengue: an update," Expert Review of Anti-Infective Therapy, vol. 10, no. 8, pp. 895907, 2012.

[4] L. H. Chen and M. E. Wilson, "Dengue and chikungunya infections in travelers," Current Opinion in Infectious Diseases, vol. 23, no. 5, pp. 438-444, 2010.

[5] L. H. Chen and M. E. Wilson, "Dengue and chikungunya in travelers: recent updates," Current Opinion in Infectious Diseases, vol. 25, no. 5, pp. 523-529, 2012.

[6] V. J. Lee, A. Chow, X. Zheng et al., "Simple clinical and laboratory predictors of Chikungunya versus dengue infections in adults," PLoS Neglected Tropical Diseases, vol. 6, no. 9, Article ID e1786, 2012.

[7] J. M. Heilman, J. de Wolff, G. M. Beards, and B. J. Basden, "Dengue fever: a Wikipedia clinical review," Open Medicine, vol. 8, no. 4, pp. e105-e115, 2014.

[8] K. Laoprasopwattana, L. Kaewjungwad, R. Jarumanokul, and A. Geater, "Differential diagnosis of chikungunya, dengue viral infection and other acute febrile illnesses in children," Pediatric Infectious Disease Journal, vol. 31, no. 5, pp. 459-463, 2012.

[9] World Health Organization, Dengue: Guidelines for Diagnosis, Treatment, Prevention and Control: New Edition, World Health Organization, Geneva, Switzerland, 2009.

[10] E. A. Hunsperger, S. Yoksan, P. Buchy et al., "Evaluation of commercially available diagnostic tests for the detection of dengue virus NS1 antigen and anti-dengue virus IgM antibody," PLoS Neglected Tropical Diseases, vol. 8, no. 10, Article ID e3171, 2014.

[11] A. Chuansumrit, W. Chaiyaratana, K. Tangnararatchakit, S. Yoksan, M. Flamand, and A. Sakuntabhai, "Dengue nonstructural protein 1 antigen in the urine as a rapid and convenient diagnostic test during the febrile stage in patients with dengue infection," Diagnostic Microbiology and Infectious Disease, vol. 71, no. 4, pp. 467-469, 2011.

[12] F. M. C. Araújo, R. S. N. Brilhante, L. P. G. Cavalcanti et al., "Detection of the dengue non-structural 1 antigen in cerebral spinal fluid samples using a commercially available enzymelinked immunosorbent assay," Journal of Virological Methods, vol. 177, no. 1, pp. 128-131, 2011.

[13] A.-C. Andries, V. Duong, C. Ngan et al., "Field evaluation and impact on clinical management of a rapid diagnostic kit that detects dengue NS1, IgM and IgG," PLoS Neglected Tropical Diseases, vol. 6, no. 12, Article ID e1993, 2012.

[14] T. R. Poloni, A. S. Oliveira, H. L. Alfonso et al., "Detection of dengue virus in saliva and urine by real time RT-PCR," Virology Journal, vol. 7, article 22, 2010.

[15] G. Yap, B. K. Sil, and L.-C. Ng, "Use of saliva for early dengue diagnosis," PLoS Neglected Tropical Diseases, vol. 5, no. 5, Article ID e1046, 2011.
[16] M. Dash, I. Mohanty, and S. Padhi, "Laboratory diagnosis of chikungunya virus: do we really need it," Indian Journal of Medical Sciences, vol. 65, no. 3, pp. 83-91, 2011.

[17] I.-C. Sam, C. L. Chua, and Y. F. Chan, "Chikungunya virus diagnosis in the developing world: a pressing need," Expert Review of Anti-Infective Therapy, vol. 9, no. 12, pp. 1089-1091, 2011.

[18] V. Reddy, V. Ravi, A. Desai, M. Parida, A. M. Powers, and B. W. Johnson, "Utility of IgM ELISA, TaqMan real-time PCR, reverse transcription PCR, and RT-LAMP assay for the diagnosis of Chikungunya fever," Journal of Medical Virology, vol. 84, no. 11, pp. 1771-1778, 2012.

[19] K. Saha, R. Firdaus, S. Chakrabarti, and P. C. Sadhukhan, "Development of rapid, sensitive one-tube duplex RT-PCR assay for specific and differential diagnosis of Chikungunya and dengue," Journal of Virological Methods, vol. 193, no. 2, pp. 521524, 2013.

[20] P. K. Dash, M. Parida, S. R. Santhosh et al., "Development and evaluation of a 1-step duplex reverse transcription polymerase chain reaction for differential diagnosis of chikungunya and dengue infection," Diagnostic Microbiology and Infectious Disease, vol. 62, no. 1, pp. 52-57, 2008.

[21] R. S. Kashyap, S. H. Morey, S. S. Ramteke et al., "Diagnosis of chikungunya fever in an Indian population by an indirect enzyme-linked immunosorbent assay protocol based on an antigen detection assay: a Prospective cohort study," Clinical and Vaccine Immunology, vol. 17, no. 2, pp. 291-297, 2010.

[22] J. Shukla, M. Khan, M. Tiwari et al., "Development and evaluation of antigen capture ELISA for early clinical diagnosis of chikungunya," Diagnostic Microbiology and Infectious Disease, vol. 65, no. 2, pp. 142-149, 2009.

[23] W. F. Wright and B. S. Pritt, "Update: the diagnosis and management of dengue virus infection in North America," Diagnostic Microbiology and Infectious Disease, vol. 73, no. 3, pp. 215-220, 2012.

[24] D. Cecilia, M. Kakade, K. Alagarasu et al., "Development of a multiplex real-time RT-PCR assay for simultaneous detection of dengue and chikungunya viruses," Archives of Virology, vol. 160, no. 1, pp. 323-327, 2015.

[25] K. Kendrick, D. Stanek, and C. Blackmore, "Notes from the field: transmission of chikungunya virus in the continental United States-Florida, 2014," Morbidity and Mortality Weekly Report, vol. 63, no. 48, p. 1137, 2014.

[26] J. G. Low, A. Ong, L. K. Tan et al., "The early clinical features of dengue in adults: challenges for early clinical diagnosis," PLoS Neglected Tropical Diseases, vol. 5, no. 5, Article ID el191, 2011.

[27] S. Patramool, E. Bernard, R. Hamel et al., "Isolation of infectious chikungunya virus and dengue virus using anionic polymercoated magnetic beads," Journal of Virological Methods, vol. 193, no. 1, pp. 55-61, 2013.

[28] T. Hirayama, Y. Mizuno, N. Takeshita et al., "Detection of dengue virus genome in urine by real-time reverse transcriptase PCR: a laboratory diagnostic method useful after disappearance of the genome in serum," Journal of Clinical Microbiology, vol. 50, no. 6, pp. 2047-2052, 2012.

[29] S. D. Blacksell, "Commercial dengue rapid diagnostic tests for point-of-care application: recent evaluations and future needs?" Journal of Biomedicine and Biotechnology, vol. 2012, Article ID 151967, 12 pages, 2012.

[30] S. Pal, A. L. Dauner, I. Mitra et al., "Evaluation of dengue NS1 antigen rapid tests and elisa kits using clinical samples," PLoS ONE, vol. 9, no. 11, Article ID el13411, 2014. 
[31] P. Pongsiri, K. Praianantathavorn, A. Theamboonlers, S. Payungporn, and Y. Poovorawan, "Multiplex real-time RTPCR for detecting chikungunya virus and dengue virus," Asian Pacific Journal of Tropical Medicine, vol. 5, no. 5, pp. 342-346, 2012.

[32] X. Lu, X. Li, Z. Mo et al., "Rapid identification of chikungunya and dengue virus by a real-time reverse transcription-loopmediated isothermal amplification method," American Journal of Tropical Medicine and Hygiene, vol. 87, no. 5, pp. 947-953, 2012.

[33] A. J. Johnson, A. J. Noga, O. Kosoy, R. S. Lanciotti, A. A. Johnson, and B. J. Biggerstaff, "Duplex microsphere-based immunoassay for detection of anti-West Nile virus and antiSt. Louis encephalitis virus immunoglobulin M antibodies," Clinical and Diagnostic Laboratory Immunology, vol. 12, no. 5, pp. 566-574, 2005. 

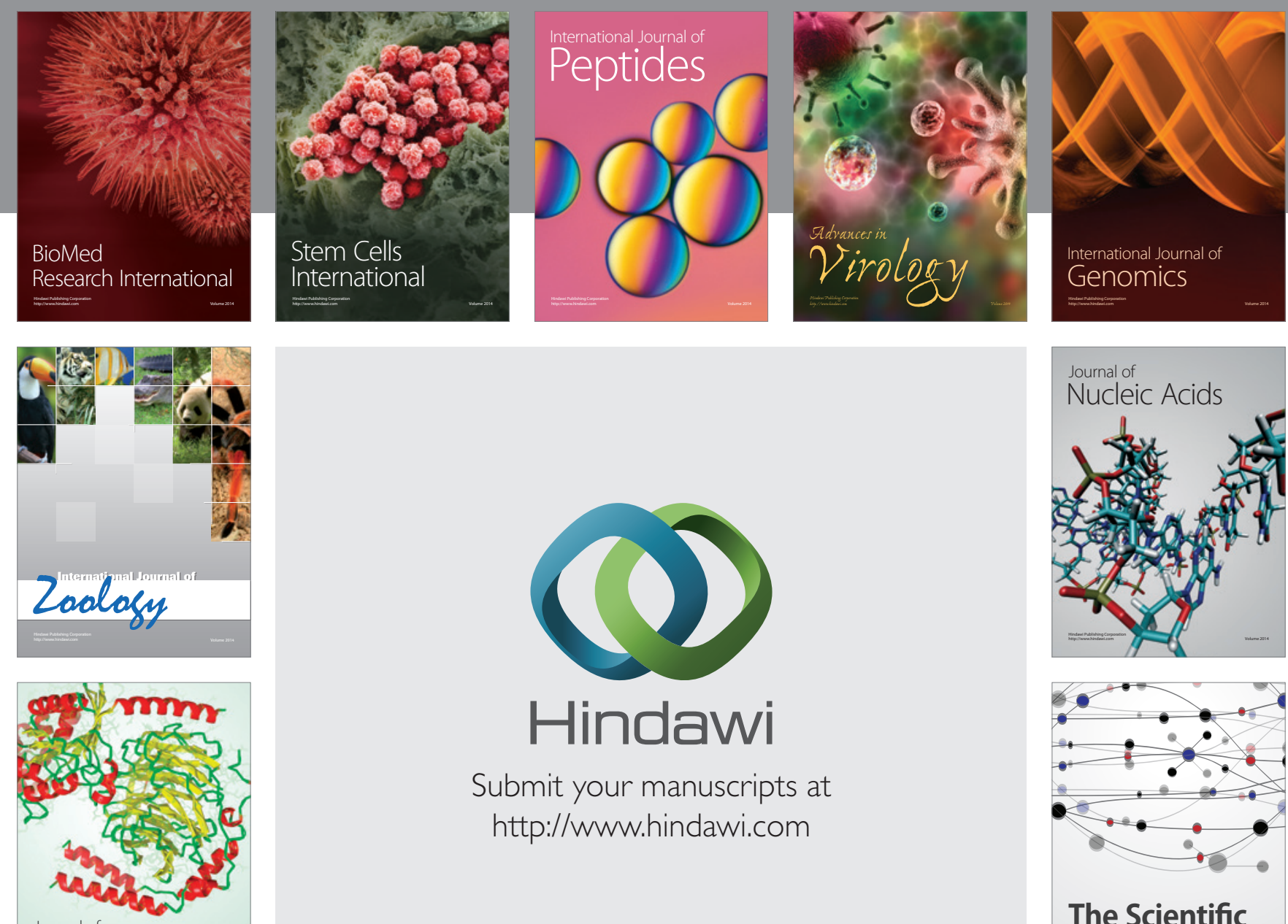

Submit your manuscripts at

http://www.hindawi.com

Journal of
Signal Transduction
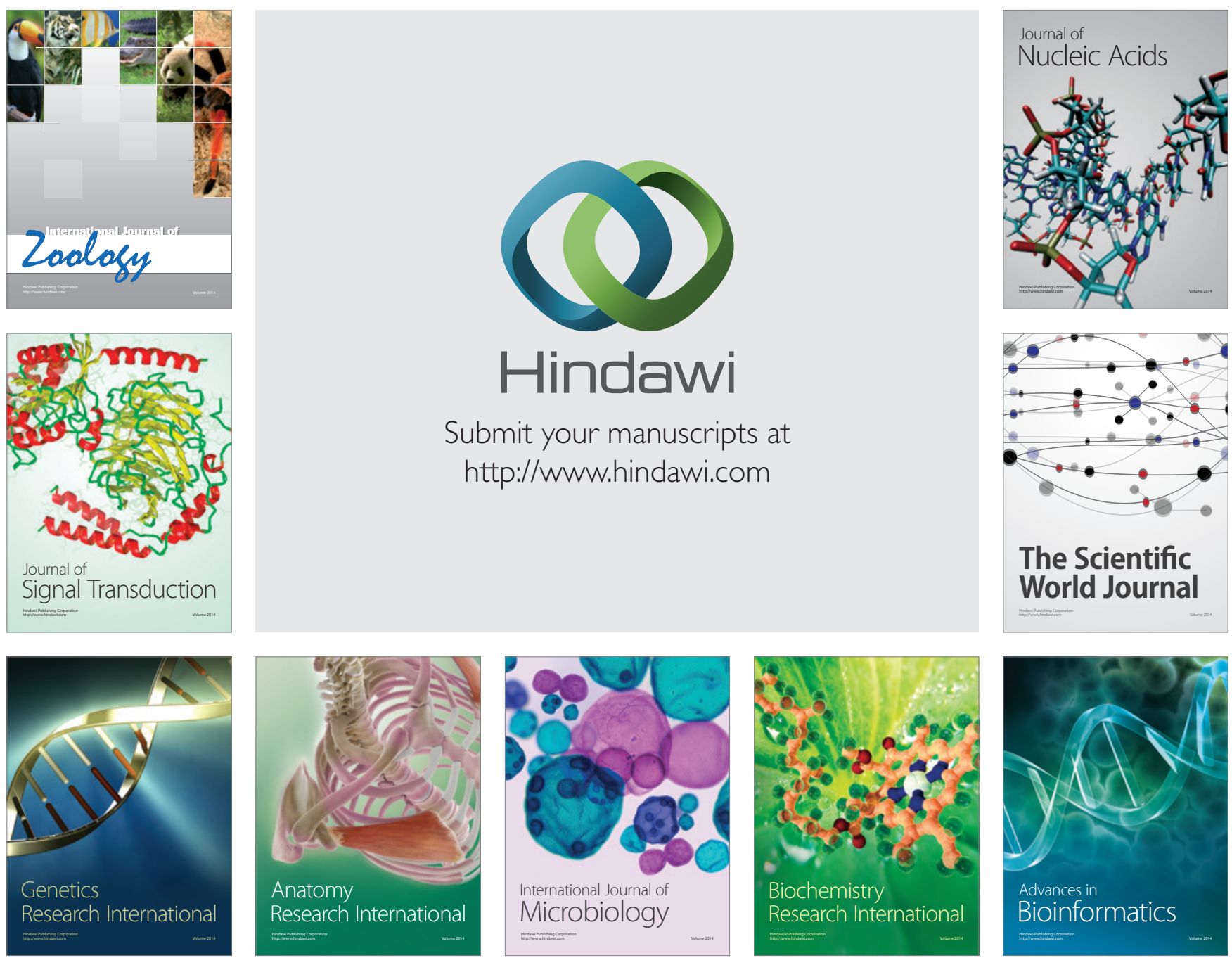

The Scientific World Journal
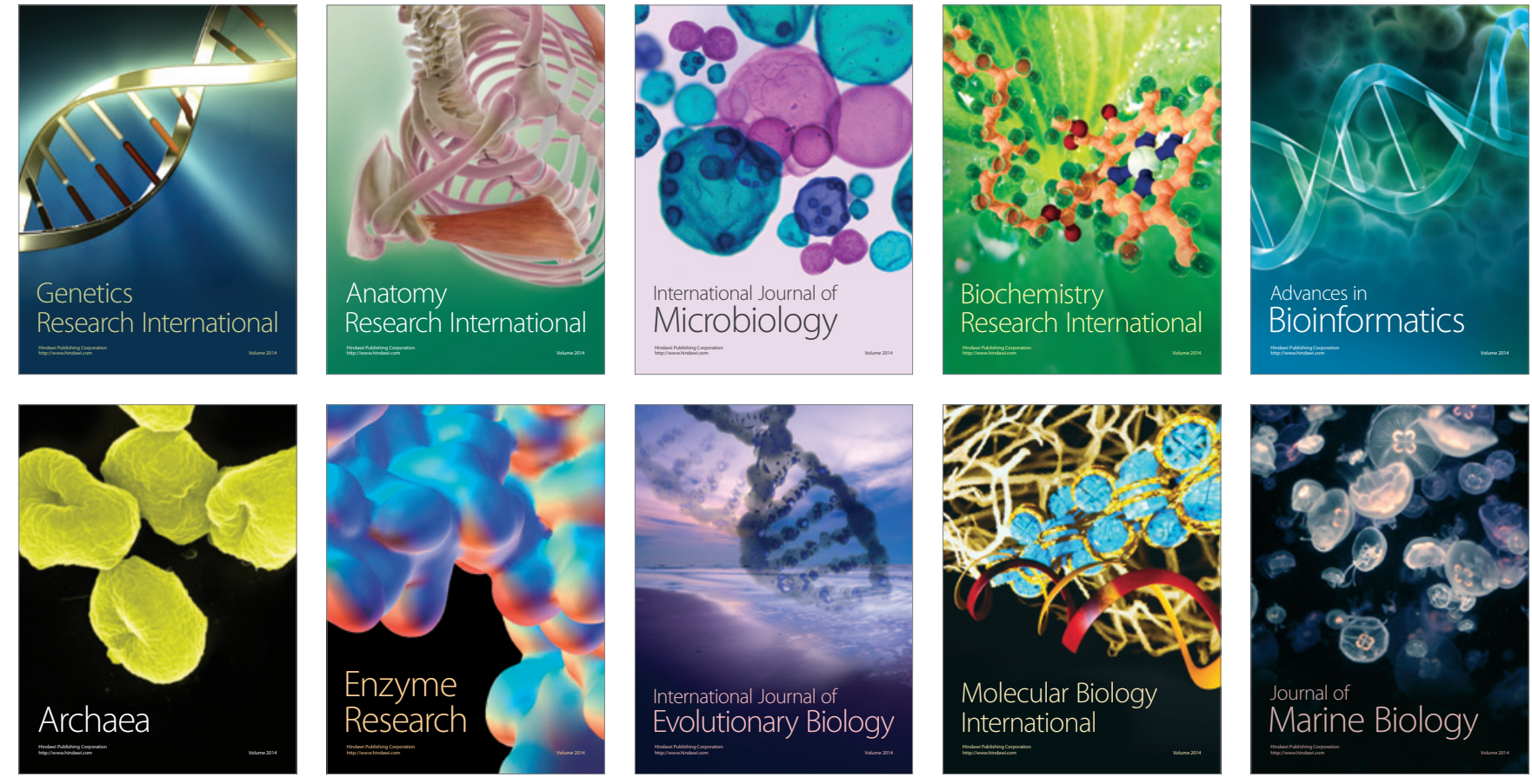